\title{
Detection of reservoir quality using Bayesian seismic inversion
}

\author{
James Gunning ${ }^{1}$ and Michael E. Glinsky ${ }^{2}$
}

\begin{abstract}
Sorting is a useful predictor for permeability. We show how to invert seismic data for a permeable rock sorting parameter by incorporating a probabilistic rock-physics model with floating grains into a Bayesian seismic inversion code that operates directly on rock-physics variables. The Bayesian prior embeds the coupling between elastic properties, porosity, and the floating-grain sorting parameter. The inversion uses likelihoods based on seismic amplitudes and a forward convolutional model to generate a posterior distribution containing refined estimates of the floating-grain parameter and its uncertainty. The posterior distribution is computed using Markov Chain Monte Carlo methods. The test cases we examine show that significant information about both sorting characteristics and porosity is available from this inversion, even in difficult cases where the contrasts with the bounding lithologies are not strong, provided the signal-to-noise ratio $(\mathrm{S} / \mathrm{N})$ of the data is favorable. These test cases show about $25 \%$ and $15 \%$ improvements in estimated standard deviations for porosity and floating-grain fraction, respectively, for peak $S / N$ of $\approx 6: 1$. The full posterior distribution of floatinggrain content is more informative, and shows enhanced separation into two clusters of clean and poorly sorted rocks. This holds true even in the more difficult test case we examine, where notably, the laminated reservoir net-to-gross is not significantly improved by the inversion process.
\end{abstract}

\section{INTRODUCTION}

Seismic data have long been highly valued as the most important information in delineating reservoir architecture and overall hydrocarbon-in-place in the oil exploration business. This is especially the case in regions where soft rock characteristics make the presence of hydrocarbons visible in reflected amplitudes. If source-rock and charge interpretations are favorable, an attractive hydrocarbon-vol- ume estimate from seismic amplitudes makes a compelling case for further appraisal work, such as drilling more appraisal wells.

But a commercial reservoir needs much more than favorable reserves - at the very least, the lithologies present must have favorable permeabilities for a commercial development to be viable. Knowledge of permeability distribution is of importance at all stages of appraisal and development. For expensive wells, e.g., deep water exploration, it is clearly important to avoid drilling low permeability areas. Another offshore scenario is that mapping of low permeability areas may provide clues to the location of unrecovered oil in high permeability areas which is unswept because of baffling from lowpermeability regions. Onshore, poorly sorted areas with lower permeability might contain appreciable bypassed oil in maturer producing fields, and thus be likely targets for infill drilling.

The value of seismic data in inferring permeability has been much more questionable, however, since flow characteristics of rocks are usually weakly coupled to their acoustic behavior in well-sorted rocks. Poor sorting is nearly always associated with reduced permeabilities, but its effect on seismic character is less well defined. The principal challenge is to see whether the rock-physics effects of poor sorting can be separated from other lithological effects that can be expected to have a broadly similar effect. The latter may be, e.g., increased volume fraction of laminated shale, increased pore volume of dispersed clay, or simply reasonable statistical variations in the rock-physics properties that can occur even if the sorting character is relatively constant. In this paper, we show that some information about sorting characteristics can be obtained from seismic data - at least for the data set under study and using a suitably calibrated rockphysics model - provided the $\mathrm{S} / \mathrm{N}$ is very good.

Two pieces of machinery are needed to address this question. The first is a suitable rock-physics model incorporating sorting effects and calibrated to the data set in question. Second, an inversion code that incorporates the generalized rock-physics model in an explicitly probabilistic framework will be necessary in order to fully explore the ambiguities in the inversion that will arise.

Texture and sorting rock-physics issues have been addressed by Marion (1990), Vernik (1997), and Dvorkin and Gutierrez (2002). A recent survey of quantitative seismic interpretation with much atten-

Manuscript received by the Editor August 10, 2006; revised manuscript received November 20, 2006; published online April 3, 2007.

${ }_{1}^{1}$ CSIRO Petroleum, Ian Wark Laboratory, Victoria, Australia, E-mail: james.gunning@ csiro.au.

${ }^{2}$ BHP Billiton Petroleum, Houston, Texas. E-mail: michael.e.glinsky@ bhpbilliton.com.

(C) 2007 Society of Exploration Geophysicists. All rights reserved. 
tion to rock-physics models, including sorting, is Avseth et al. (2005). For this paper, we use the theory of DeMartini and Glinsky (2006), which has much in common with the shaley-sand Yin-Marion model (Marion, 1990; Avseth et al., 2005), excepting the nonsupporting secondary material are lithic fragments, not shale. For our purposes, a theory covering only the grain-supported range of poorly sorted rocks is required, without diagenetic effects: A much broader theory covering other types of textures as well can be found in Dvorkin and Gutierrez (2002). Vernik (1997) pays particular attention to diagenesis and loading issues, developing hybrid theoretical-empirical expressions from simple effective medium theories for pores of particular shapes. The lithic-fragment effects described by the DeMartini and Glinsky (2006) model are broadly similar to what Avseth et al. (2005) call sedimentation-controlled effects, in contrast to the broad regional trends applicable to our data, which are usually understood to be diagenetically controlled (in this case by compaction). Another valuable study on texture, sorting, and seismic inversion is Bachrach and Mukerji (2004), but this has a major focus on poorly consolidated sediments, in contrast to our data.

The Bayesian seismic inversion code Delivery that we use to implement the model is described in Gunning and Glinsky (2004). Other papers of a similar Bayesian spirit have appeared, e.g., Buland et al. (2003), Buland and Omre (2003), and Eidsvik et al. (2004), but the associated codes are not publicly accessible. The open-source license for Delivery makes the results of our research more easily accessible and reproducible for the geophysics community. Other methods have appeared (Coleou et al., 2005) with a strong emphasis — like ours — on inverting directly to petrophysical variables and using rock-physics models for stabilization, but with a strong emphasis on optimization via annealing, the full uncertainty of the inversion is not available.

Bayesian methods have also been used to try and discriminate poorly sorted lithologies from seismic data (Avseth et al., 2001). Like our work, the approach in Avseth et al. (2001) is explicitly probabilistic, integrating rock-physics models, Gassman-style fluid effects, and AVO techniques in a Bayesian framework, but one of the major limitations is that the informative seismic amplitudes in the Bayesian classification are taken directly from seismic volumes. The results are thus vulnerable to tuning effects, and the uncertainties also do not reflect the increased ambiguity which occurs in these cases. More recent work (e. g., Dutta et al., 2006) attempts to integrate sequence-stratigraphic considerations into the inversion for rockquality parameters along similar lines, but the same limitation regarding amplitudes holds. We use a forward convolutional model in the inversion process, which implicitly deconvolves the effects of tuning, a widely known benefit (e.g., Abrahamsen et al., 1997). To our knowledge, our work constitutes the first attempt to infer sorting behavior in a Bayesian framework that models the interference effects associated with limited-resolution wavelets.

We are interested in inverting for sorting behavior in a depth range and provenance identical to that of the data used to calibrate the rock-physics model of DeMartini and Glinsky (2006). The data are from a turbidite system (of significant commercial interest) with a source that is rich in lithic fragment material. The depositional environment is somewhat unusual in that the poorer-sorted areas are not characterized by a marked increase in the volume fraction of dispersed clay (it is only a few percent), in contrast to many poorer-sorted rocks, where the finer nonclay materials typically settle out with much dispersed clay. More detail on the mineralogical issues can be found in DeMartini and Glinsky (2006). In the general case, one would expect to incorporate dispersed clay content into the rockphysics model, but this is not required for these data. A rich discussion of sorting issues in various depositional environments can be found in McManus et al. (1988) and Boggs (2006); for example, poorer-sorted sands are usually observed in the more distal parts of the deposition, or in proximal areas where rapid decrease in flow velocity occurs, e.g., fan deltas.

The outline of this paper runs as follows. We revisit in Rock-Physics Models the floating grain model of DeMartini and Glinsky (2006), as a summary for the reader and to establish some notation. We present in The Inversion Model a brief explanation of how this model is incorporated into the Bayesian inversion code, explaining what the parameterization is, the effective media assumptions, and the details of the forward model. In Numerical Examples, we illustrate the implications of this model for two inversion problems strongly inspired by the geology of the data set under study. Example A: A simple model system, examines the simplest seal over reservoir toy problem, and explores the characteristics of the prior in some detail. In Example B, more complex model based on field data, a much more fully fledged model incorporating a complex seal structure and two reservoirs is developed. Some of the more subtle questions concerning the rock-physics model and our findings are addressed in the Discussion, and we summarize in the Conclusions.

\section{ROCK-PHYSICS MODELS}

We recapitulate briefly the rock-physics model described in DeMartini and Glinsky (2006). The purpose of this section is not to evangelize this particular model contra other models in the literature, but to summarize the chosen theory and establish some notations and ideas for the convenience of readers. Of the material following, only that matter concerning the separation of the effects of sorting from "conventional" variability using cluster analysis is new - we believe this is a useful supplement to the original DeMartini and Glinsky (2006) paper. We emphasize that the data set is unusual in having low dispersed-clay content for a poorly sorted sandstone, and that the theory is applicable to the grain-supported regime.

The DeMartini and Glinsky model assumes measurements apply in the Gassmann low-frequency limit, and that the reservoir is a homogeneous isotropic medium. In general, they distinguish between the fluid porosity $\phi$ and structural porosity $\phi_{s}$. If the grain density and bulk modulus are $\rho_{g}$ and $K_{g}$, respectively, then filling the pore space with a fluid or suspension with properties $\rho_{f}, K_{f}$ produces an effective medium of density

$$
\rho=\rho_{g}(1-\phi)+\rho_{f} \phi
$$

and compressional and shear velocities

$$
\begin{aligned}
& v_{p}^{2}=\frac{K_{g}}{\rho}\left(\frac{3\left(1-\nu_{m}\right)}{1+\nu_{m}} \beta+\frac{(1-\beta)^{2}}{\phi_{s}\left(K_{g} / K_{f}-1\right)+1-\beta}\right), \\
& v_{s}^{2}=\frac{K_{g}}{\rho} \frac{3\left(1-2 \nu_{m}\right)}{2\left(1+\nu_{m}\right)} \beta .
\end{aligned}
$$

Here the matrix bulk modulus is $K_{m}$, the dimensionless matrix bulk modulus is $\beta=K_{m} / K_{g}$, and $\nu_{m}$ is the matrix Poisson's ratio. The dependence of the matrix $\beta$ on (structural) porosity is taken to be that of a conventional critical-porosity model (Nur et al., 1991; Mavoko et al., 1998) 


$$
\beta\left(\phi_{s}\right)=\left(1-\phi_{s} / \phi_{c}\right)^{\lambda}
$$

where $\phi_{c}$ is a critical suspension porosity, usually around 0.42 , and $\lambda$ a data-fitted constant.

For a poorly sorted collection of grains, the finer grains are treated as a secondary component, which contributes in two pieces: (1) some small volume fraction of fine grains do not support the rock matrix and act like a pore-space fluid, whereas (2) the remaining fraction is bound or captured into the load-bearing frame as the rock is buried over time. If the overall fraction of small grains introduced is $f_{*}$, and a fraction $f_{c}$ of these are captured, then $\phi_{\mathrm{flt}} \equiv\left(1-f_{c}\right) f_{*}$ is the volume fraction of floating grains. This floating fraction is treated as an effective fluid and modeled via Gassman substitution, whereas the effect of the captured grains is absorbed by using the structural porosity $\phi_{s}=\phi+\phi_{\mathrm{flt}}$ in $\beta$, equation 4 , and the load bearing or structural porosity appearing in the denominator of equation 2. The effect of the bound grains on the matrix Poisson's ratio can be shown to be weak, and is thus neglected.

DeMartini and Glinsky provide extensive arguments as to why treating the second component as a significantly different mineral does not yield an adequate match to the experimental data shown below. Core and thin-section analysis of the data in question also precludes the presence of dispersed or finely laminated clay. The data are then modeled using a bimodal (large and small floating grains) mixture of a single mineral, which has the advantage that the overall $\mathrm{P}$-wave velocity simplifies to the expression

$$
\begin{aligned}
v_{p}\left(\phi, \phi_{\mathrm{flt}}\right)^{2}= & \frac{K_{g}}{\rho_{g}(1-\phi)+\rho_{f} \phi}\left[\frac{3\left(1-\nu_{m}\right)}{1+\nu_{m}} \beta\left(\phi+\phi_{\mathrm{flt}}\right)\right. \\
& \left.+\frac{\left(1-\beta\left(\phi+\phi_{\mathrm{flt}}\right)\right)^{2}}{\phi\left(\frac{K_{g}}{K_{f}}-1\right)+1-\beta\left(\phi+\phi_{\mathrm{flt}}\right)}\right],
\end{aligned}
$$

where $\beta\left(\phi+\phi_{\mathrm{flt}}\right)$ is equation 4 evaluated at the modified porosity $\phi_{s} \leftarrow \phi+\phi_{\mathrm{flt}}$. Arguments are also furnished to demonstrate that the $v_{s}$ versus $v_{p}$ regional trend does not change to leading order under this model, a prediction which is corroborated by the data.

The DeMartini and Glinsky $\left\{v_{p, i}, \phi_{i}\right\}$ data ( $i$ runs over samples) from log measurements in the province of interest appear to fall into distinct clusters. The measurements are restricted to short, well-resolved clean portions of the log, arithmetically averaged, and fluidsubstituted to a reference brine. One cluster, which may be depthcorrelated with core measurements of decent permeability $\left(\approx 10^{2}\right.$ $-10^{3} \mathrm{mD}$ ) and fairly monodisperse sands, is modeled as clean rock, with no floating component $\left(\phi_{\mathrm{flt}}=0\right)$. This $\log$ data are used in a nonlinear regression $v_{i}=v_{p}\left(\phi_{i}, \phi_{\mathrm{flt}}=0, \lambda\right)+\epsilon_{p, i}$ for the exponent $\lambda$, assuming generic values for quartz $\left(K_{g}=37 \mathrm{GPa}, \rho_{g}=2.65 \mathrm{~g} /\right.$ $\left.\mathrm{cm}^{3}\right)$, brine $\left(\rho_{f}=1.05 \mathrm{~g} / \mathrm{cm}^{3}, v_{p, f}=1850 \mathrm{~m} / \mathrm{s}\right)$, a mid-porosity range typical clean sand Poisson's ratio $\left(\nu_{m}=0.15\right)$, and a critical porosity $\phi_{c}=0.42$. The regressed fit of $\hat{\lambda}=1.724$ is then used in a second regression for $\phi_{\mathrm{flt}}$, using only the anomalous data, yielding $\hat{\phi}_{\mathrm{flt}}=0.039$, which is a plausible average value for the anomalous data. The data and fits are shown in Figure 1. From this regression, we speculate that the anomalous data are probably a mixture of rocks with variable sorting characteristics, ranging from nearly clean to perhaps $\phi_{\mathrm{flt}}=5 \%$, judging from the regressed value $\hat{\phi}_{\mathrm{flt}}=0.039$ and the scatter about the regression.
Because the floating fraction is an unobserved quantity for each measurement, an important statistical question is how its distribution can be disentangled from the regression variance $\epsilon_{p}$. The underlying distribution of $\phi_{\mathrm{flt}}$ is unknown, but is likely to contain clusters, often coinciding with well groups. We have chosen to fix the variance of $\epsilon_{p}$ to that of the regional trend, which accounts for the dispersion in the velocities due to conventional effects, and attribute the remaining dispersion to the effect of $\phi_{\mathrm{flt}}$. A multicluster analysis (using a modified form of MCLUST [Fraley and Raftery, 2003]) of the univariate distribution of the residuals $\xi_{i}=v_{p, i}-v_{\text {reg.trend }}\left(\phi_{i}\right)$, with cluster 1 fixed to the regional error (mean 0 , variance $\operatorname{var}(\xi)$ $=\sigma_{v}^{2}$,reg. ) shows the most statistically significant clustering model is a two-cluster split. The data points grouped with the regional trend are shown circled in Figure 1.

Linearization of the best fit velocity relation $v_{p}\left(\phi, \phi_{\mathrm{flt}}\right)$ (equation 5) about a suitable mean porosity $\bar{\phi} \approx 0.24$ and $\bar{\phi}_{\text {flt }}=0$ yields a straight line approximation

$$
v_{p}=a_{p}+b_{p}\left(\phi_{\mathrm{flt}}+(1+g) \phi\right)+\epsilon_{p}
$$

where we have written the three required constants in this way for consistency with the notation of DeMartini and Glinsky (2006). The (zero mean) error term is $\epsilon_{p}$. The linearization is clearly reasonable for the data clusters of Figure 1.

In DeMartini and Glinsky (2006), the response to loading is captured using a standard exponential regression model dependent on the effective stress $\sigma_{\text {eff }}$, with an additional term describing the grain capturing effect:

$$
\phi=a_{\phi}+b_{\phi}\left(1-e^{-\sigma_{\mathrm{eff}} / P_{0}}\right)-\frac{\phi_{\mathrm{flt}}}{1-f_{c}}+\epsilon_{\phi}
$$

This expression is a statement that the total space occupied by the pore fluid and the finer grains is compressed under loading in a conventional way, and amounts to a definition of the capture fraction $f_{c}$. Basin modeling provides estimates of the effective stress, and the floating fraction estimates from the velocity regression on data clusters then provide a way to estimate $a_{\phi}$ and $f_{c}$ through fitting. When this process is performed on the data set here, estimates of $f_{c}=$ $0.3522, a_{\phi}=1.1, b_{\phi}=-0.8759, P_{0}=5.52 \mathrm{MPa}$, and $\sigma_{\epsilon_{\phi}}=0.0024$ are produced.

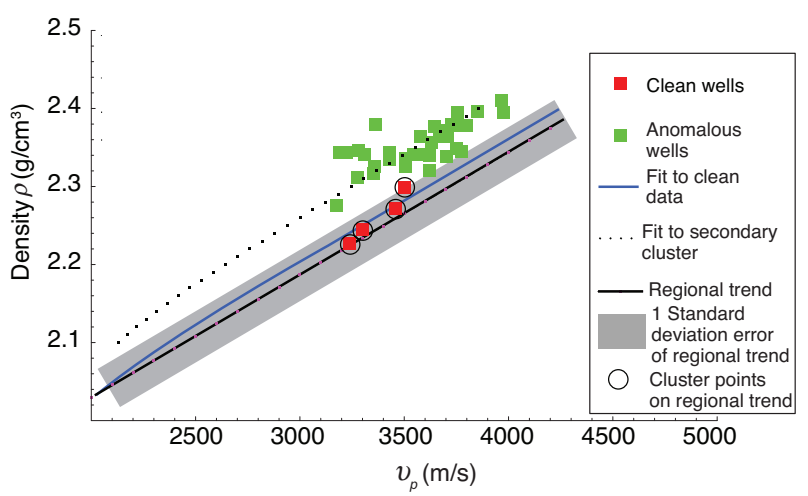

Figure 1. Density versus $v_{p} \log$ data for clean sands and anomalous sands, together with nonlinear regression best fits $\left(\phi_{\mathrm{flt}}=0,0.039\right.$, respectively, $\lambda=1.724$ in both cases). The straight line regional trend is the average across a much larger data set for the region, with approximate errors as shown. 
This model exhibits all the usual characteristics of poorly sorted trend models. In particular, a plot of $v_{p}\left(\phi, \phi_{s}-\phi\right)$ versus $\phi$ (using equation 5), corresponding to varying sorting with fixed structural porosity $\phi_{s}$, shows the usual shallow-slope sorting-trend behavior, comparable with, for example, Figure 1.11 of Avseth et al. (2005).

\section{THE INVERSION MODEL}

The inversion code we use operates on a layer-based model with effective-media approximations for the layer properties, a 1D forward convolutional model, linearized rock-physics trend models, and approximate Zoeppritz relations for the AVO reflectivity equations. The layer-top times are the fundamental geometric variables. Each layer is modeled as a finely laminated mixture of a reservoir end member and a nonreservoir end member. The nonreservoir end member internal properties are $v_{p}, v_{s}$, and $\rho$. The reservoir end member internal properties are $v_{p}, v_{s}, \phi$, and $\phi_{\mathrm{flt}}$. The net-to-gross (NG) is the volume fraction of reservoir end member appearing as fine-scale horizontal laminations within a particular layer (i.e., has no relation to the internal composition of the reservoir rock). Within the reservoir end member, the pore-fluid type, saturation, density, and $v_{p}$ are possible additional variables.

Geometrically, the moving layer boundaries make this a simple example of a marked-point model. The effective-media laws used in each layer are, successively, (1) Ruess averaging for pore-scale fluid mixing in the reservoir end member, (2) Gassmann's law for fluid substitution in the reservoir end member, and (3) Backus averaging for laminated sand/shale upscaling using the NG. This produces a set of effective layer properties for each layer. Synthetic seismic traces are then generated from the effective layer properties, with reflections placed at the layer boundaries, using the convolutional model and linearized Zoeppritz equations. The reflectivity is taken as a second-order (in incidence angle $\theta$ ) approximation to the (isotropic) Zoeppritz reflectivity $R_{p p}$ of layer 1 above layer 2, namely

$$
R_{p p}=\frac{1}{2}\left(\frac{\Delta \rho}{\rho}+\frac{\Delta v_{p}}{v_{p}}\right)+\theta^{2}\left[\frac{\Delta v_{p}}{2 v_{p}}-\frac{2 v_{s}^{2}\left(\frac{\Delta \rho}{\rho}+\frac{2 \Delta v_{s}}{v_{s}}\right)}{v_{p}^{2}}\right],
$$

with $\rho=\left(\rho_{1}+\rho_{2}\right) / 2, v_{p}=\left(v_{p, 1}+v_{p, 2}\right) / 2, v_{s}=\left(v_{s, 1}+v_{s, 2}\right) / 2, \Delta \rho$ $=\rho_{2}-\rho_{1}, \Delta v_{p}=v_{p, 2}-v_{p, 1}$, and $\Delta v_{s}=v_{s, 2}-v_{s, 1}$. Note that the inversion operates directly on primary rock-physics parameters (layer times, velocities, densities, porosities, saturations, etc.), so the impedances and reflectivities merely come along for the ride. Hydrocarbon-in-place estimates will be driven by the thickness (i.e., $v_{p}$ and times), NG, and porosity, whereas permeability estimates will have a contribution from $\phi_{\mathrm{flt}}$.

The Bayesian framework correctly accounts for all the requisite uncertainties in the rock physics and stratigraphic geometry in realistic models (within the limitations of the linearized rock-physics model, the Gaussian noise assumptions, etc.). The rock-physics models constitute the prior specification for the rock properties, and interpreted horizons with (usually generous) error bars form the prior for the layer times. Prior distributions of NG, fluid content, and saturations are established by consultation with the geologist, petrophysicist, etc. Quantities that are physically limited in range (e.g., 0 $\leq \mathrm{NG} \leq 1)$ are given truncated-Gaussian prior distributions, which we notate as, e.g., $\mathrm{NG} \sim N_{\mathrm{TR}}\left(\mu, \sigma^{2}\right)$. The probability weight associ- ated with the Gaussian tails outside the permissible range is collapsed onto the endpoint, so these distributions are a mixture of a spike(s) and a smooth Gaussian part.

The inversion code uses a Markov Chain Monte Carlo algorithm to generate a suite of stochastic models from the posterior distribution, which fully embody the solution to the inverse problem. Interrogation of this posterior ensemble produces (among other things) best estimates of the rock properties (means, medians), and the associated uncertainty (covariances, histograms, etc.).

This software has an established style of representing loading or compaction curves where P-wave velocity is regressed directly against suitable loading terms. The naturally augmented version of these regressions suitable for incorporating sorting effects is

$$
\begin{gathered}
v_{p}=A_{v_{p}}+B_{v_{p}} d+D_{v_{p}} \phi_{\mathrm{flt}}+\epsilon_{p}^{\prime}, \\
\phi=A_{\phi}+B_{\phi} v_{p}+C_{\phi} \phi_{\mathrm{flt}}+\epsilon_{\phi}^{\prime},
\end{gathered}
$$

using the existing notation in Gunning and Glinsky (2004) (d = loading depth: the LFIV term in that paper is suppressed). The shear relation is unchanged. The fully linear form of the prior and the assumption of Gaussian regression errors also enables a multi-Gaussian prior to be formulated. For each reservoir layer, this prior has the conditional form $P\left(v_{s} \mid v_{p}\right) P\left(\phi \mid v_{p}, \phi_{\mathrm{flt}}\right) P\left(v_{p} \mid d, \phi_{\mathrm{flt}}\right)$, where the variance of each of these Gaussian components comes from the regression variance, e.g., $\operatorname{var}\left(\epsilon_{p}^{\prime}\right)=\sigma_{p}^{2}$. The effective-stress dependence can be effected by taking the loading depth $d \equiv 1-\exp \left(-\sigma_{\text {eff }} / P_{0}\right)$ if required.

Details of how the DeMartini and Glinsky form of the regressions (equations 6 and 7) are coerced into these forms (i.e., equations 9 and 10) are given in Appendix A.

\section{NUMERICAL EXAMPLES}

We present here two examples illustrating how the inversion works using a floating-grain model fitted to data from the province of interest. There are two pay zones of interest. The first example is the standard test problem of a single isolated reflector (focusing on the upper pay), and the second is a more complex model based on the full $\log$ data and both pay zones. The region of interest contains various lithologies, but the main cap rock above the oil-bearing sands is a shale, so we are chiefly interested in the trend properties of the pay sand and this overlying shale.

We are interested also in the value of near- and far-offset seismic data. Since the reflectivity (equation 8 ) is only $O\left(\theta^{2}\right)$, we do not consider very-far-offset (e.g., $55^{\circ}$ ) data in deference to this limitation: far stacks are about $30^{\circ}$ in these studies. Similar caveats are applied in codes that use, e.g., the Shuey approximation (Avseth et al., 2005). Naturally, the shear velocity $v_{s}$ contributes more substantially to the reflectivity for the far stack. The far stack amplitudes will thus help to constrain the $v_{s}$ parameters via the reflectivity (equation 8), hopefully in a complementary fashion to the constraints on $v_{s}$ that are embedded in the rock-physics prior model.

The data that informs these examples creates what is conventionally called a class IIp AVO effect (Avseth et al., 2005) for the upper sands (polarity flip occurs between near and far stacks), which is the subject of the first example. The lower sands have more like a class I effect, but in both cases the reflection coefficients are relatively weak. The shifting of classes is caused by the increasing separation of the impedance trends with depth. In the absence of floating grains, 
both events look like class I anomalies, but the reflections are still relatively weak. It is always worthwhile remembering that the AVO classifications are somewhat uncertain because of the seismic noise, and that the classification has no explicit role in the inversion formalism.

\section{Rock trends for sands and shales}

There are no major trending discontinuities expected in the data set associated with tectonic, overpressuring, or diagenetic effects. We would expect the trends to be of utility only in basin-local prediction, as is common in calibrated rock-physics models. The clean sand trends are not dissimilar to published trends for the same basin. The (brine-saturated) shale and sand trends are common to both examples, so we dispense with these first. Quoted errors are one standard deviation.

\section{Shales}

Standard shale trends for the area in question are, with $z$ as depth below mudline,

$$
\begin{aligned}
& v_{p}=-1640+0.946 z(\mathrm{~m}) \pm 145(\mathrm{~m} / \mathrm{s}), \\
& \rho=0.650 v_{p}^{0.166} \pm 0.029\left(\mathrm{~g} / \mathrm{cm}^{3}\right), \\
& v_{s}=-1027+0.801 v_{p} \pm 63(\mathrm{~m} / \mathrm{s}) .
\end{aligned}
$$

\section{Sands}

For the $v_{p}$ relation 5 , the clean trend applicable is obtained by a linearization of the fit line shown in Figure 1 (the nonlinear fit to the clean rock data cluster), whose maximum-likelihood fit has $\lambda$ $=1.724$ and material constants as per the accompanying description. At the mean data porosity $\bar{\phi}=0.24$, the linearization of equation 5 to the form equation 6 yields constants $a_{p}=5729 \mathrm{~m} / \mathrm{s}, \quad b_{p}=$ $-9440 \mathrm{~m} / \mathrm{s}$, and $g=0.0248$, with estimated error $\sigma_{p}=\sqrt{\operatorname{var}\left(\epsilon_{p}\right)}$ $=229 \mathrm{~m} / \mathrm{s}$.

The loading trend for the pay sands is established in DeMartini and Glinsky (2006) as

$$
\phi=1.1-0.876 Z_{\mathrm{eff}}-1.54 \phi_{\mathrm{flt}}+\epsilon_{\phi},
$$

with $Z_{\text {eff }} \equiv\left(1-e^{-\sigma_{\text {eff }} / 5.52 \mathrm{MPa}}\right)$ and error $\sigma_{\phi}$ $=\sqrt{\operatorname{var}\left(\epsilon_{\phi}\right)}=0.0024$. Since the shale trends are against $z$, we have converted this stress regression (equation 14) to a depth trend, since the loading term $Z_{\text {eff }}$ is very nearly linear in depth over the depth range of interest. The equivalent pay sand depth trend (cf. equation 7) is

$$
\begin{aligned}
\phi= & 0.526-5.25 \times 10^{-5} z(\mathrm{~m}) \\
& -1.54 \phi_{\mathrm{flt}}+\epsilon_{\phi} .
\end{aligned}
$$

The last regression coefficient $(1.54=1 /(1$ $\left.-f_{c}\right)$ ) corresponds to the capture fraction $f_{c}$ $=0.35$. With the understanding that $z$ now plays the role of $Z_{\mathrm{eff}}$, the conversion formulas of Appendix A yield the constants $A_{v_{p}}=645 \mathrm{~m} / \mathrm{s}, B_{v_{p}}$ $=0.508, \quad D_{v_{p}}=5494 \mathrm{~m} / \mathrm{s}, \quad A_{\phi}=0.592, \quad B_{\phi}=$ $-1.03 \times 10^{-4} \mathrm{~s} / \mathrm{m}$, and $C_{\phi}=-0.976$.
The shear trend for the data regressed directly to yield

$$
v_{s}=-1218+0.894 v_{p} \pm 69(\mathrm{~m} / \mathrm{s}) .
$$

We have used the clean-sand regional-trend error estimates $\sigma_{\epsilon_{\phi}}^{\prime}$ $=0.0093$ and $\sigma_{\epsilon_{p}}^{\prime} \approx 105 \mathrm{~m} / \mathrm{s}$ in the augmented model, corresponding to the conventional-effects assumption described earlier.

\section{Example A: A simple model system}

The aim here is to determine whether the presence of floating grain material in the reservoir rock is ascertainable from reflected amplitudes using the rock-physics model and regional regressions just derived. We begin with the simplest two-layer shale/sand system, which is free of the complication of interference or tuning effects.

The well logs used to construct the prior have some clean rocks (no floating grains) and rocks with floating content of around $2 \%$ to $5 \%$. To model the inferability of the float fraction, we constructed synthetic seismic truth-case stacks for near- and far-stack angles of a few degrees and about $30^{\circ}$, using a truth-case model with $5 \%$ float and $\mathrm{NG}=1$, and all other parameters at the most likely values from the trends. The reservoir fluid is taken as brine for this simple study. Figure 2 illustrates the system, with truth-case plus posterior near and far synthetics from the posterior of case (3) we describe below.

For inversion, the prior on floating fraction in the sand fraction of layer 2 is taken as $N_{\mathrm{TR}}\left(0,0.05^{2}\right)$, so the truth case is one standard deviation away from the prior mean, and the prior is biased towards clean sands. We attempt to compute the posterior floating fraction from three cases where the amount of laminated shale in layer 2 varies. (1) No interbedded shale allowed; the prior on NG in layer 2 is $N_{\text {TR }}(1,0)$ (which forces $\mathrm{NG}=1$ ), with near-stack only seismic. (2) Some interbedded shale permissible; the prior is $\mathrm{NG} \sim N_{\mathrm{TR}}\left(1,0.2^{2}\right)$ (roughly $60 \%$ to $100 \%$ sand), with near-stack seismic only. (3) The same prior $\mathrm{NG} \sim N_{\mathrm{TR}}\left(1,0.2^{2}\right)$, but with both near and far stacks. (Remember the inversion code shifts the probability associated with $\mathrm{NG}>1$ on to the endpoint $\mathrm{NG}=1$, so the prior in cases 2 and 3 is a 50:50 mixture of clean and laminated sands.) Recall that the NG is associated with subseismic shale laminations (not dispersed clay), and the floating fraction is associated with the sandstone lamina. For these rocks, increasing layer NG and floating-grain content tend to pull the upscaled rock properties in approximately similar directions.
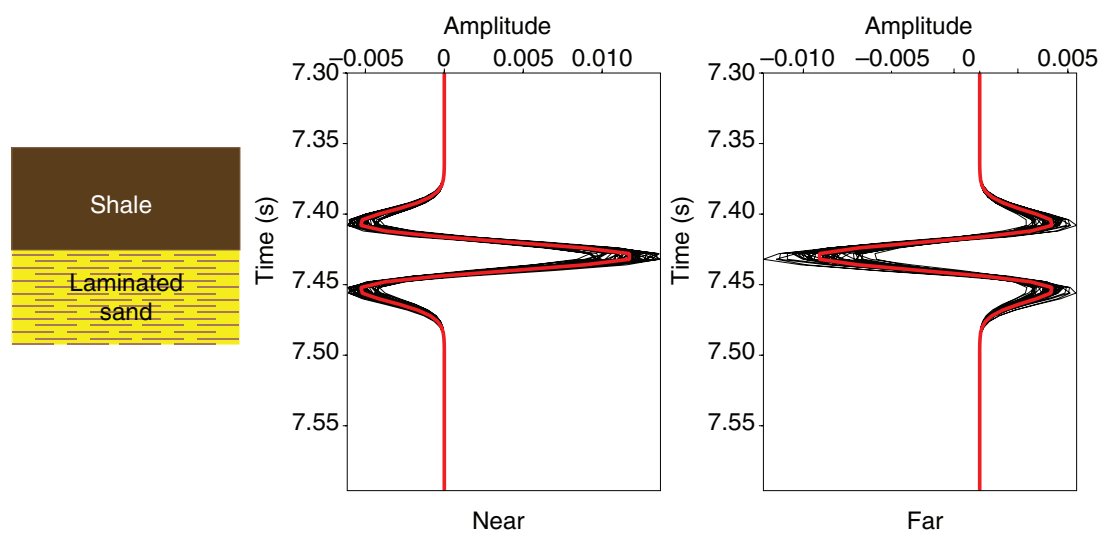

Figure 2. Two-layer model system with truth-case (thick red) seismic traces and synthetics from the posterior (black) for the inversion case 3 described in the text. The absolute noise level is set at 0.002 for both stacks. 
For case 1, Figures 3-5 show three possible forms of the prior, varied for illustrative purposes, on which we superpose the original log data, which illustrates how the floating-grain effect smears out the regional prior. In Figure 3, the model prior is constructed with artificially narrow $\sigma_{\phi}=0.002$, showing how the parameters arise from a clear 50:50 mixture of clean-rock regression points and an elliptical smear from the effects of the floating grains. The clean rock trend is obviously far too narrow to embrace the clean well measurements, but the clean trend is clearly visible and centered. Recall that the well data is from a spread of depths, so it is not expected that the prior (applicable at the reservoir depth only) covers all well data. The difference between the general effects of increasing loading and increasing floating-grain content is shown. Clearly, a convolution of this mixture distribution along the depth direction would cover all the well data comfortably.

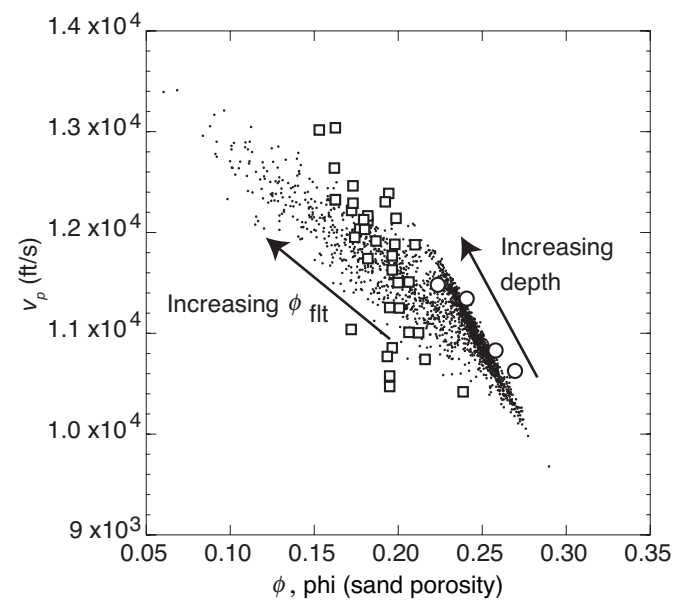

Figure 3. This and Figures 4 and 5 show three pedagogic priors for velocity versus porosity in a pure sand layer at the reservoir depth. Clean well data points (circles) and float-polluted well data points (squares) are plotted on all three graphs; dots $(\cdot)$ are draws from the model prior.

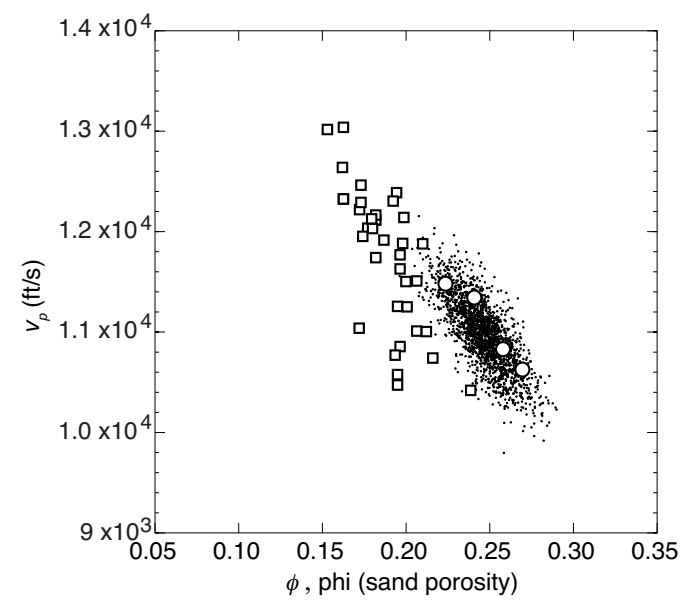

Figure 4. Second illustrative prior: prior drawn from clean rocks only (float fraction $\phi_{\mathrm{flt}} \sim N_{\mathrm{TR}}(0,0)$ ), with broad porosity uncertainty $\sigma_{\phi}=0.02$. The tails of the distribution do not contain the floatinggrain data comfortably. Clean well data points (circles), float-polluted well data points (squares); dots (.) are draws from model prior.

\section{Inversion analysis of posteriors}

Fixed NG, single (near) stack.-Figures 6 and 7 show salient scatter plots of properties of the sand layer before and after inversion. Figure 8 shows the comparison of the floating-grain distribution. The peak $\mathrm{S} / \mathrm{N}$ is set at about $6: 1$. These and subsequent figures

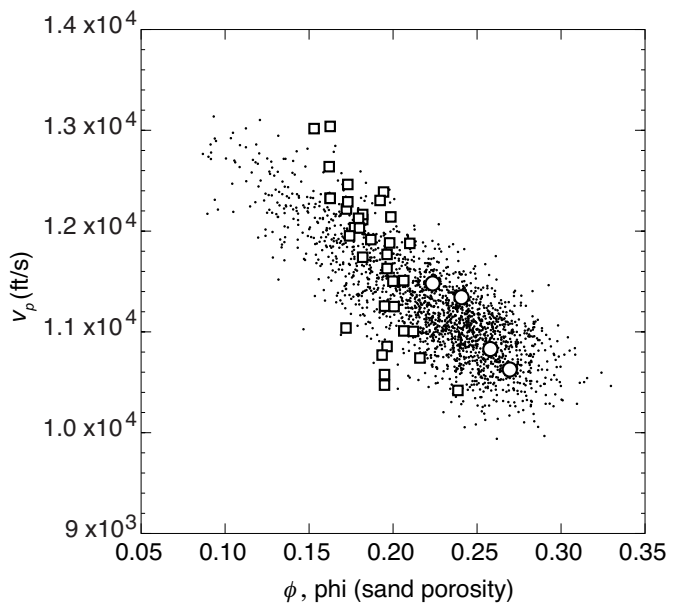

Figure 5. Third illustrative prior: prior with $\sigma_{\phi}=0.02$ and float fraction $\phi_{\text {flt }}$ distributed as $\sim N_{\mathrm{TR}}\left(0,0.05^{2}\right)$. Only a few measurements appear to lie at the periphery of the distribution, but the mixture character is not as clear to the eye as in Figure 3. Clean well data points (circles), float-polluted well data points (squares); dots (.) are draws from model prior.
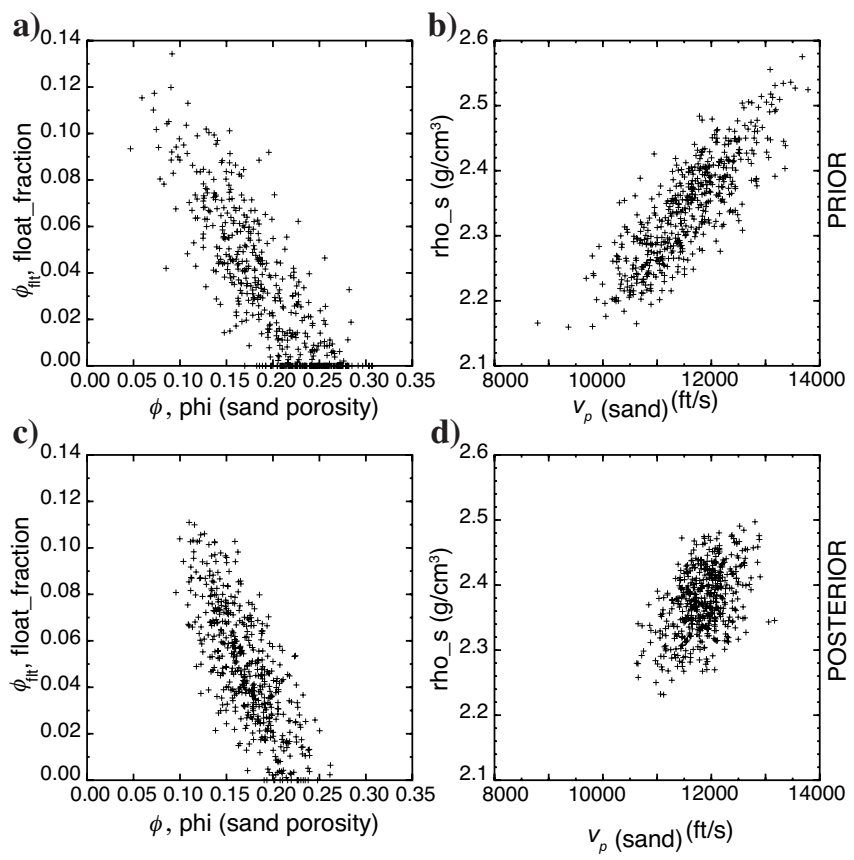

Figure 6. This and Figure 7 show scatterplots from prior distribution ( $\mathrm{a}$ and $\mathrm{b}$ ) and posterior ( $\mathrm{c}$ and d) for example A (2-layer model with sand below shale), fixed $\mathrm{NG}=1$, near stack only. (a and $\mathrm{c}$ ) float_fraction $\left(\phi_{\mathrm{flt}}\right)$ versus sand porosity. A perceptible narrowing around the true answer of $\phi_{\mathrm{flt}}=5 \%$ is visible: Fewer clean sands are produced in the posterior (see also the prior and posterior histograms of $\phi_{\mathrm{flt}}$ in Figure 8). (b) and (d) The sand density versus sand velocity $v_{p \text { (sand) }}$ scatterplot narrows more obviously. 
use symbols defined in Gunning and Glinsky (2004) as follows: (1) $R_{\text {near }}$ and $R_{\text {far }}$, defined as $R_{p p}$ for the near and far stack, from equation 22 ; (2) overall layer effective density $\rho_{\text {eff }}$ and velocity $v_{\text {peff }}$, defined in equations 9 and 10 of that paper. The inversion is clearly able to detect the presence of floating grain material and refine the porosity estimates (see Figure 8).

Free NG, single (near) stack.-Figure 9 shows salient scatter plots of properties of the sand layer after inversion, where the model has additional $\mathrm{NG}$ freedom in the prior $\mathrm{NG} \sim N_{\mathrm{TR}}\left(1,0.2^{2}\right)$. As shown in Figure $9 \mathrm{f}$, the inversion produces virtually no posterior sharpening of the NG distribution. If anything, the NG broadens slightly, perhaps reflecting the ambiguity between shale lamina and floating grains (using only normal-incidence reflectivity). Nevertheless, the floating-grain fraction estimate is still noticeably improved, as is the porosity (green histogram, Figure 9d and e).

Free NG, near and far stack.- Inversion using far-offset data in principle may help narrow down floating grain porosities better, as the far-offset stack carries additional information about the shear velocity via equation 8 . The far stack for this test case is set at about $30^{\circ}$ (cf. a few degrees for the near) and the reflected amplitude is much weaker (AVO effects). The noise level was set at the same value as for the near stack.

For this case, it turns out that the far stack eliminates the weak broadening of the NG estimates (blue histogram, Figure 9f), and increases the fraction of realizations with appreciable floating grain content by about $50 \%$ (blue curve, Figure $9 \mathrm{~d}$ ). The porosity estimate
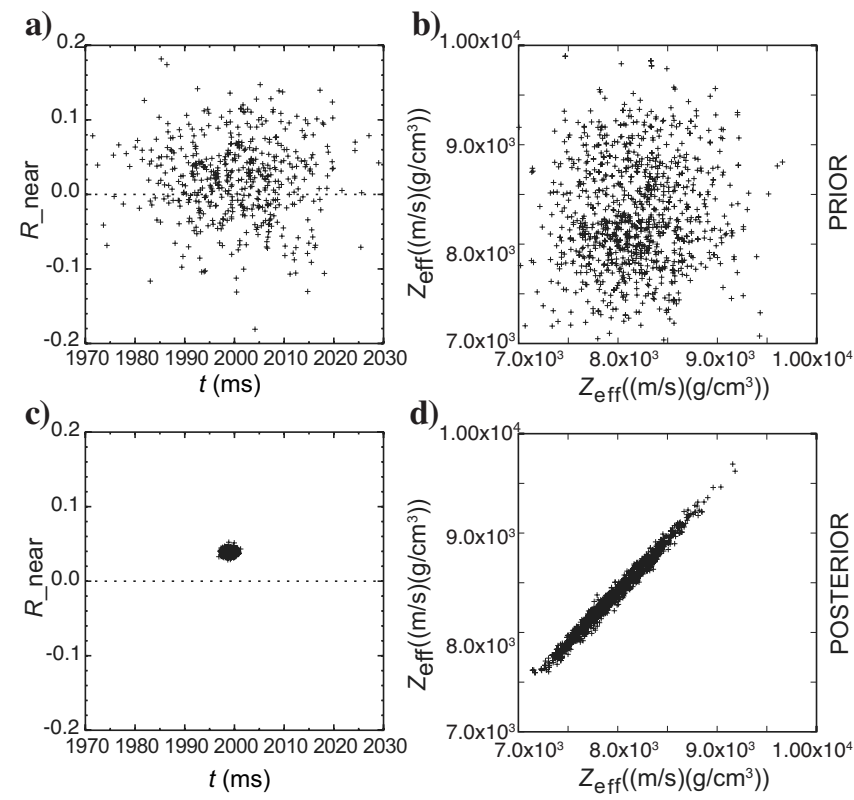

Figure 7. More scatterplots from prior distribution ( $a$ and $b$ ) and posterior ( $\mathrm{c}$ and d) for example A (2-layer model with sand below shale), fixed NG $=1$, near stack only. (a) and (c) The effective reflection coefficient $R_{\text {near }}$ versus layer time $t$ is clearly pinned down sharply. As usual, these parameters are most heavily constrained. (b) and (d) The layer-1 versus layer-2-P wave impedance. Very strong correlations between these are induced, but the marginal distribution of each is more weakly constrained. is also noticeably sharpened around the truth-case value, and the fraction of clean sands (high porosity, low floating-grain content) is markedly decreased (Figure 9e). A significant sharpening in the shear velocities $v_{s}$ and especially the overall far-reflection coefficient $R_{-}$far also occurs.

In summary, the test case here appears to show that an inversion is capable of detecting the presence or absence of floating grain material for the kinds of rocks studied at the depths of interest, as well as tune up the reservoir porosity estimate. Addition of far stack data appears to help resolve the ambiguity between NG effects and floating grain content, and improves the detectability of poorly sorted rocks by around $50 \%$. The basic reflection coefficients are relatively weak $(|R| \sim 0.03)$ for both near and far stacks at this depth, so we regard this as a significant result for an obviously difficult test case. In contrast to our usual experience of Bayesian inversion with imaged seismic data, the updates to the sand/shale NG are very weak, despite the encouraging results for floating-grain fraction. This phenomenon is a particular conspiracy of the impedance trends for the rocks in question, so this asset appears to be a particularly challenging example. Further challenges arise in the more complex field example of the next section.

\section{Example B: More complex model based on field data}

In this example, based on actual field data, the complicating effects of additional lithologies and tuning considerations come into play. The oil reservoir system we model here features upper and lower pay sands which are capped by a complex draping structure including thick, acoustically hard marls and thin, soft, silty layers. An overall simplified six-layer sequence for the system has been modeled as (1) marl, (2) silt-marl stringer complex, (3) shale, (4) upper sand, (5) shale, and (6) lower sand, where the silt-marl stringer complex is an upscaled (impermeable) layer absorbing some of the very thin structures in the cap. The near-offset reflectivity from the marl edge is sufficiently strong to dominate the reflection from the upper sand top, so much extra information comes from the interface with the shale below, and an additional $30^{\circ}$ stack whose P-wave reflectivity is more comparable to the marl. The wavelet used in producing the synthetics is a $15.6 \mathrm{~Hz}$ Ricker-2, $w(t) \sim\left(1-2 \pi^{2} t^{2} f_{p}^{2}\right)$ $\times \exp \left(-\pi^{2} t^{2} f_{p}^{2}\right)$.

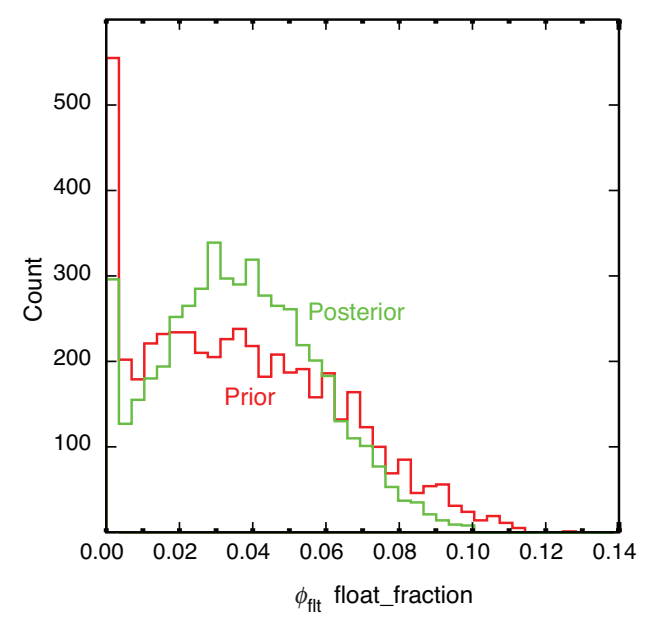

Figure 8. Prior and posterior histograms of $\phi_{\mathrm{flt}}$ in layer 2 of simple sand-shale problem (example A, fixed $\mathrm{NG}=1$, near stack only). 
Figure 10 shows the upscaled layer structure superposed on the logs and shale plus sand trends for the model. Note there are distinct trends for the marl and stringer complex.

The main pay sands are not very clean, and are estimated to have NG values of around $65 \pm 10 \%$ (on the seismic scale). Transitions between shale lamina and sand are quite distinct. As with all discretized models of multiscale natural systems, there is some art in blocking the well logs so the coarse-scale layers used in the inversion have a sensible interpretation of the NG parameter. The layer time-thicknesses are also selected to be seismically resolvable, and are rarely less than $1 / 6$ of a wavelength. We define NG from coarselayer vertical averages based on conventional facies classification algorithms. The forward model uses Backus averaging to handle subseismic layering effects, so the NG parameter should be regarded as a layer-scale parameter; within-layer geologic heterogeneities are effectively absorbed into the NG.

Oil is proved in these reservoirs with saturations of around $60 \%$. For simplicity, we fix the oil probability (set to 1) and saturation (set to 0.6$)$ in these layers. The floating-grain fraction in the main reservoirs is given a prior of $N_{\mathrm{TR}}\left(0.02,0.03^{2}\right)$, which gives a significant prior probability to the zero-float or clean-sand case. The "truth case" corresponds to a float fraction of $3.5 \%$. Note that the prior in these inversions is sufficiently loose to allow layers 1,2 , or 3 to pinch out.

Figure 11 shows some typical "spaghetti plots" of the synthetic seismic from the posterior plotted against the truth-case data, for both stacks. Typical realizations from prior and posterior are also shown as "layer-cake" images of layers against realization number.

As might be expected, the inversion produces strong updates to parameters like the layer times, impedances, effective layer velocity, and porosity. Some salient results are shown in Figures 12 and 13: In general the most significant improvements occur in the thicker layers $(4,6)$, whereas tuning effects significantly limit what may be inferred about the thinner structures. A reasonable improvement in the float-fraction estimate occurs, in particular the fact that the posterior significantly reduces the zero float possibility. The most likely prediction is correct at around 0.035 . The NG estimates are barely improved, mainly because the upper sand offers a weak impedance contrast to its mixing shale when floating grain material is present at around 3\% (the trend curves). The sensitivity to floating-grain frac- a)

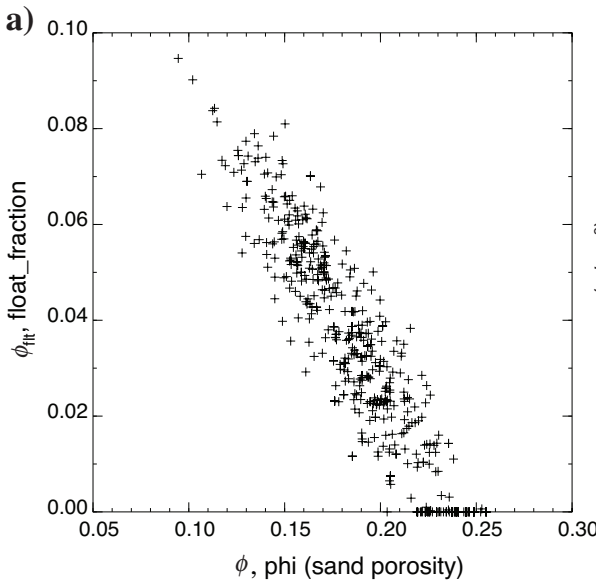

d)

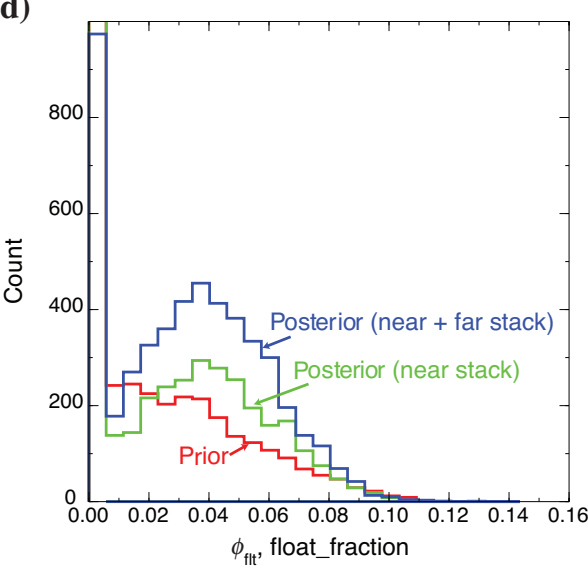

b)

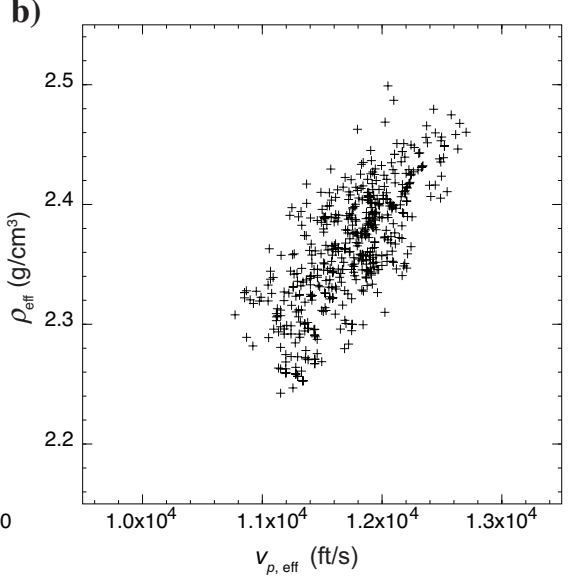

e)

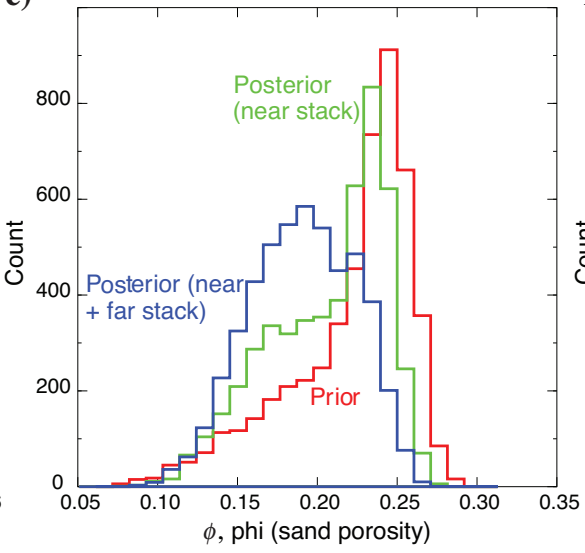

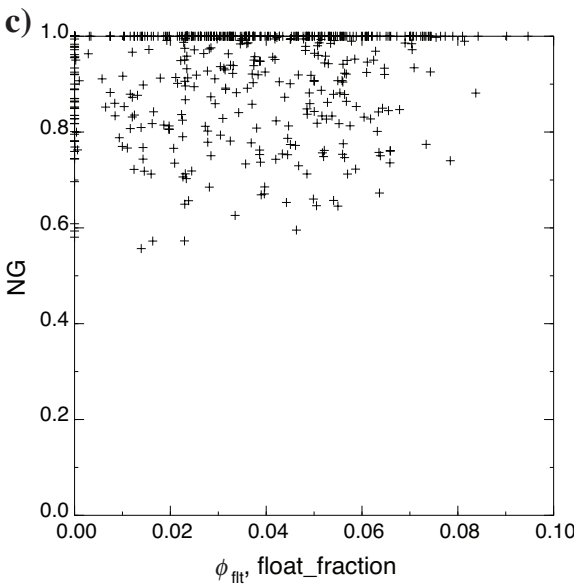

f)

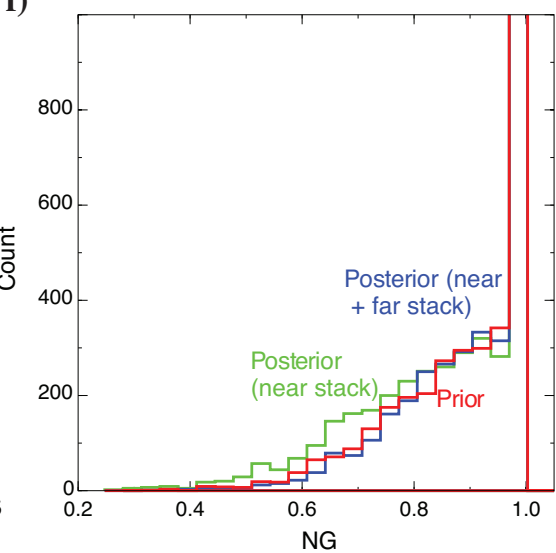

Figure 9. Example A results. Scatterplots from the posterior for the model with looser NG distribution in prior (near stack only). (a) Floatinggrain fraction $\phi_{\mathrm{flt}}$ versus sand porosity $\phi$, (b) effective density $\rho_{\text {eff }}$ versus effective velocity $\left(v_{p, \text { eff }}\right)$. (c) Scatterplot of NG versus $\phi_{\mathrm{flt}}$. No obvious strong correlation between NG and $\phi_{\mathrm{flt}}$ appears here, with the density strongest near the truth-case values $\left(\phi_{\mathrm{flt}}=0.05, \mathrm{NG}=1.0\right)$. (d) Histogram of $\phi_{\mathrm{flt}}$ from the prior (red) and posterior, using only near stack (green) and also both stacks (blue). Similar histograms of porosity shown in (e) and NG in (f). The truth case porosity is $\phi=0.17$. 
tion is very much higher than that to NG, so the update is stronger. Again, the far-stack data helps to refine the shear properties significantly, but this does not couple back through the prior strongly enough to improve estimates of the quantities of direct interest $\left(\phi_{\mathrm{flt}}\right.$, $\phi, \mathrm{NG})$ markedly. Though not shown, the far-stack data greatly improves the $v_{p}$ statistics of the shale in layer 5, but not the adjoining reservoir sands. Overall, however, the improvements over the prior are not strong in view of the aggressive $\mathrm{S} / \mathrm{N}$.
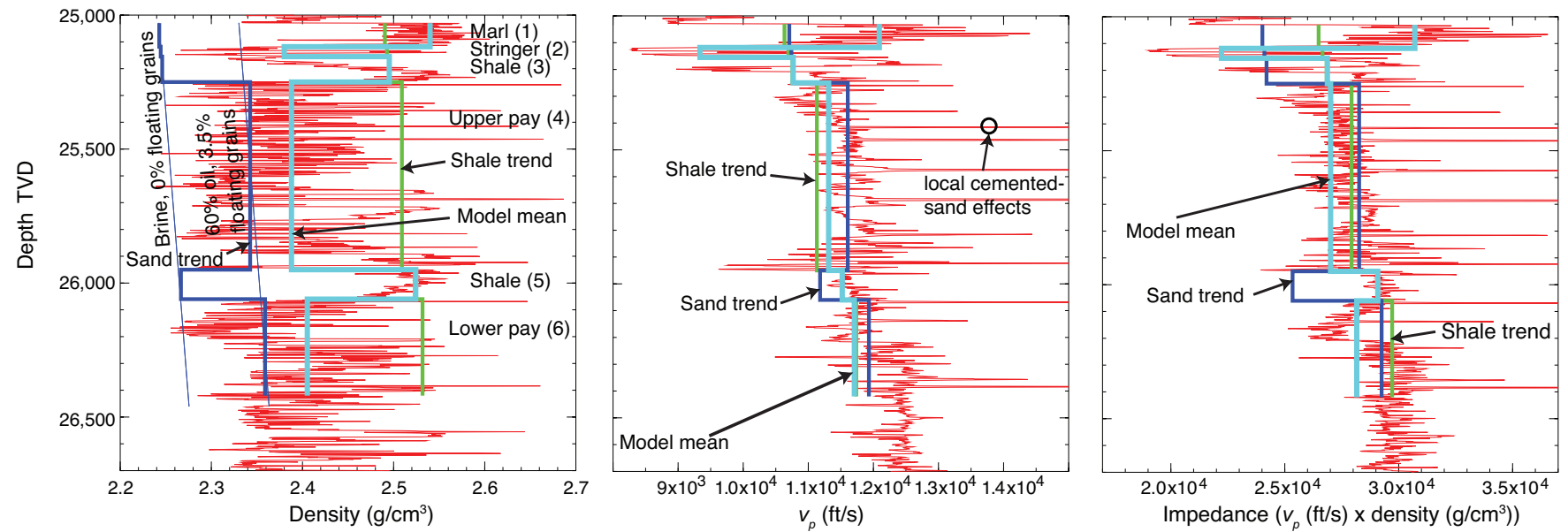

Figure 10. Example B: Layer-based model truth-case properties for the test model: logs (red) and truth-case mean properties (cyan) are shown, for density, P-wave velocity and normal impedance. The succession of six layers is clearly evident. The shale trend is shown green, sand is blue: The deflections in the sand trend lines are due to the floating-grain term, and these are shown without fluid substitution. The streaks in the logs are caused by small cemented sand units that have no large-scale effect on the seismic.
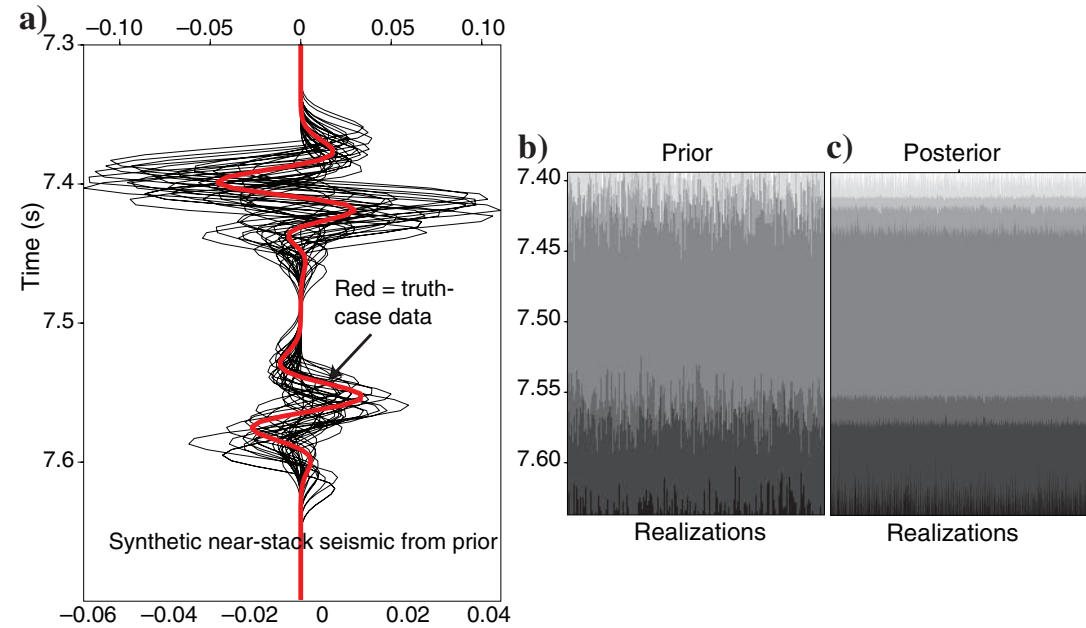

Figure 11. Inversion results for the six-layer example B model shown in Figure 10. (a, d, e). Synthetic seismic curves (black) for model samples, superposed on actual data (red), as drawn from prior (a, near stack), and posterior (d, near stack; $\mathrm{e}$, far stack). Note the noise level is set very low; the $\mathrm{S} / \mathrm{N}$ is at least 10:1. (b and c) Approximately 50-layer realizations from the prior and posterior, respectively, in time. The layers are shaded in ascending grayscale according to layer number.
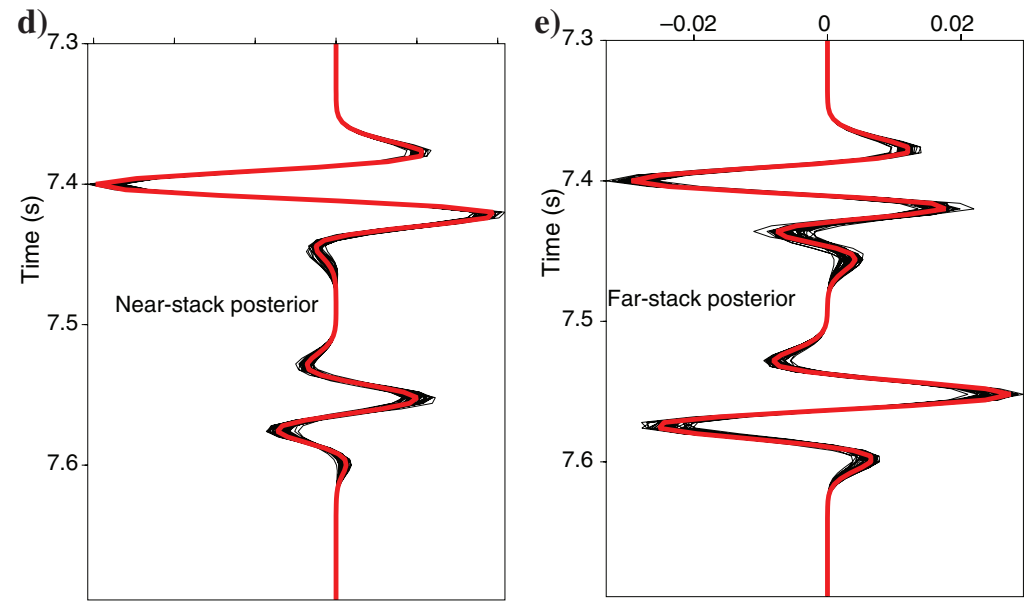
In conclusion, the more realistic toy problem here shows that detection of poorly sorted material is possible with very favorable $\mathrm{S} / \mathrm{N}$. Several asset-specific issues make this more difficult than might at first be expected. The first is that the reflection at the top of the upper pay falls in the sidelobe of the very strong reflection from the overlying marl layer, so tuning effects and uncertainties in the modeling of the cap rock package, in general, limit what may be discerned about the underlying sand. Secondly, the particular loading behavior characteristic of the pay depth makes the shale impedance quite close to that of the sand, so the overall strength of the main reservoir reflection is notably weaker than other nearby events. In view of the difficulties, it is relatively consoling that positive information about the floating-grain contribution can be drawn when very little can be said about the NG.

\section{DISCUSSION}

\section{Rock-physics model}

It could be argued that the effects we seek to model warrant a more sophisticated model of the effects of sorting than that of DeMartini and Glinsky (2006). However, for the regime in which we seek to make predictions, we believe this theory to be adequate for our purposes, well fitted to the data, and statistically significant. We note that a common feature of all the rock-physics models cited in the In- troduction is the need for empirical relations or constants at some point in the theory, whether at the naive level of the critical-porosity power exponents of DeMartini and Glinsky (2006), critical concentrations in Dvorkin and Gutierrez (2002), the deeper Hertz-Mindlin plus coordination-number theory in Dvorkin and Nur (1996), or the pore-shape factor versus porosity relations of Vernik (1997). Over the ranges we seek to predict, the rock-physics effects are close to linear, and thus it is virtually certain that any credible model using a single parameter to represent sorting will linearize to the forms of equations 6 and 7 . The only likely ornament is a weak effect on the shear velocity - weak enough to fit within the $v_{p}$ versus $v_{s}$ regression error already established for the regional trend (see also the discussion in DeMartini and Glinsky, 2006). Moreover, to paraphrase a remark of G. Mavko, probably any appropriate theory chosen from the literature and well fitted to the data will produce similar predictions.

\section{Common characteristics of the inversion examples}

Some of the more interesting information available from the inversion requires higher-order statistical expressions than simple means and standard deviations. In particular, since many of the distributions are skewed or truncated, examination of histograms or bi- a)

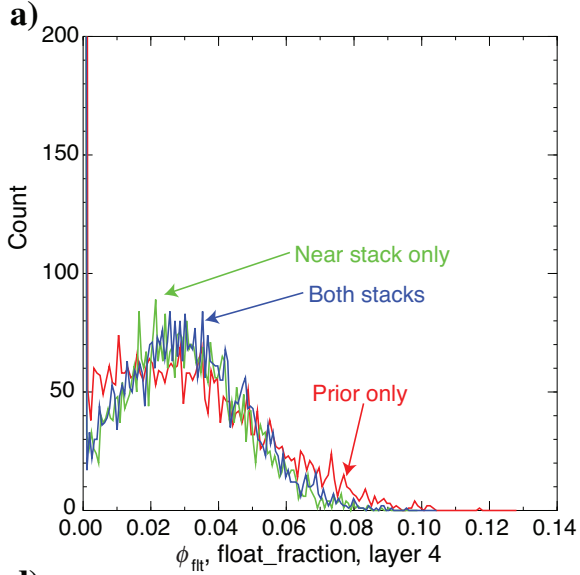

d)

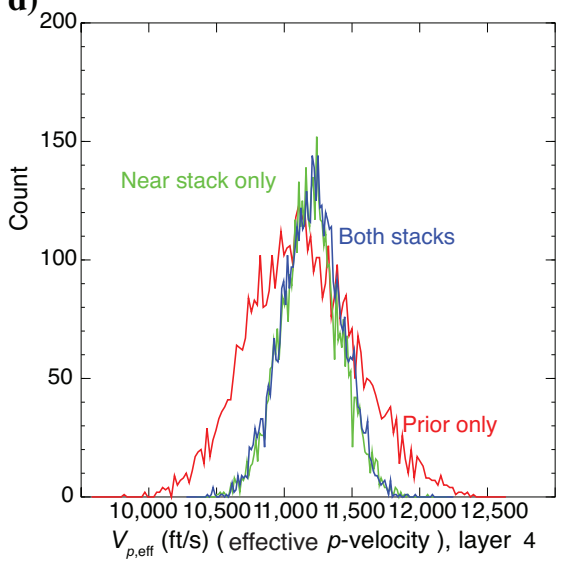

b)

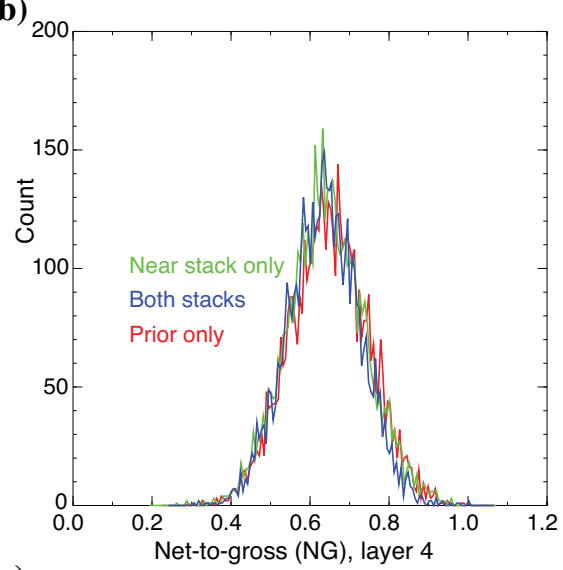

e)

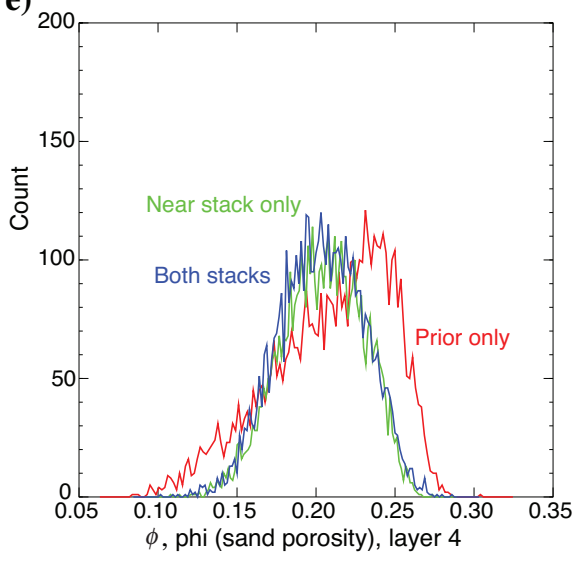

c)

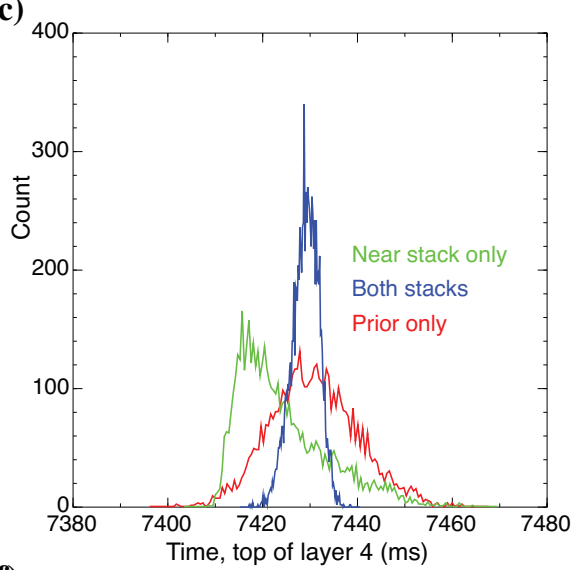

f)

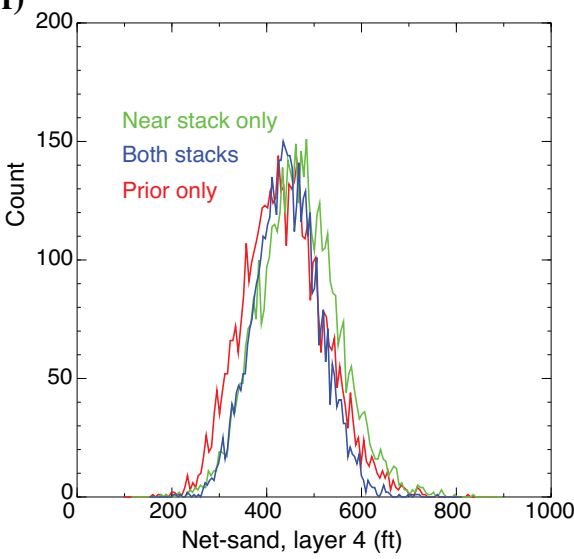

Figure 12. Prior (red), near-stack-only posterior (green), and both-stack posterior (blue) histograms for selected properties of the upper sand (layer 4). The strange asymmetric layer-4 time posterior in (c) for near stack only (green) is evidence that the top layering structure is not well resolved from this stack alone. More revealing detail appears in the scatterplots of Figure 13. 
variate scatter plots is more revealing than simple means and standard deviations.

For example, we show in Table 1 selected properties of the reservoir layer ranked by the degree which the standard deviation is reduced by the inversion, for the two-stack simple interface example A. The rather modest improvement on the floating fraction estimate in this table does not fully convey the significant shift in the univariate distribution shown in Figure 8 or $9 \mathrm{~d}$.

Clearly from Table 1, as one would expect, the geometry (layer times) and (between-layer) reflectivities are the best-identified parameters in the inversion. Any within-layer property per se is much less well identified, including the $\mathrm{P}$-wave impedance. Improvements in the estimation of effective density, fluid porosity, and floatinggrain fraction are all roughly comparable, but less than the P-wave impedance update. In this particular environment, the identification of NG is very problematic, but this is (partly) due to the shale-sand trend crossover phenomenon, as remarked previously.

As far as univariate statistics go, the same generic findings and ranking hold for the more complicated six-layer model example $\mathrm{B}$. For bivariate statistics, in both examples, very strong correlations between the impedances of adjacent layers are produced (e.g., Figure $7 \mathrm{~d}$, coinciding with the sharply defined reflection coefficient), but the univariate posterior distributions of any individual layer impedance are nowhere near as sharp. Perhaps counterintuitively, no strong correlations appear between NG and parameters like porosity or $\phi_{\mathrm{flt}}$ in the posterior distributions. This is largely because the individual layer impedances are not especially well pinned down, but also because the reservoir layer impedance does not correlate strongly with NG (at this depth). Since the narrowing of the layer imped- ance is obviously an upper bound on what may be inferred about any particular rock property within a layer, the modest findings of Table 1 are no surprise.

The fundamental limitations on inference are set by the parameters that control the overall statistical spread in the layer impedance. These come down ultimately to the regression errors in fitting the regional trends, and it should be clear that tight characterization of the nonreservoir facies is equally important to that of the reservoir rocks. Figure 7d, for example, illustrates clearly that the prior rockphysics uncertainties in impedance are much greater than the relative impedance change commanded by the seismic amplitudes (for this particular data set). In terms of posterior sharpening, the sorting characterization parameter $\phi_{\mathrm{flt}}$ seems to fare not much worse than other classical rock parameters like the $\mathrm{P}$-wave velocity and porosity, if we make the judgment on the standard deviation alone. A rough and ready argument corroborating the results of Table 1 is this: using realizations from the prior distribution of example A, case 2, the $\mathrm{P}$-wave impedance regresses directly against $v_{p}, \phi, \phi_{\mathrm{flt}}$, or NG with Pearson $r^{2}$ values of of $0.95,0.88,0.71$, and 0.03 , respectively. This is exactly the relative ordering of the standard deviation improvement we see, with roughly the same quality of improvement. Again, the improvement in $\phi_{\mathrm{flt}}$ is best understood in terms of the refinement of the posterior histograms, as per Figures 8, 9c, and 12a.

A likely and commonsense generalization is that the inference improvement for any parameter (including sorting) is the improvement of the impedance multiplied by the fraction of the variance of the impedance accounted for by that parameter. This generic principle should hold true for other data sets and environments.
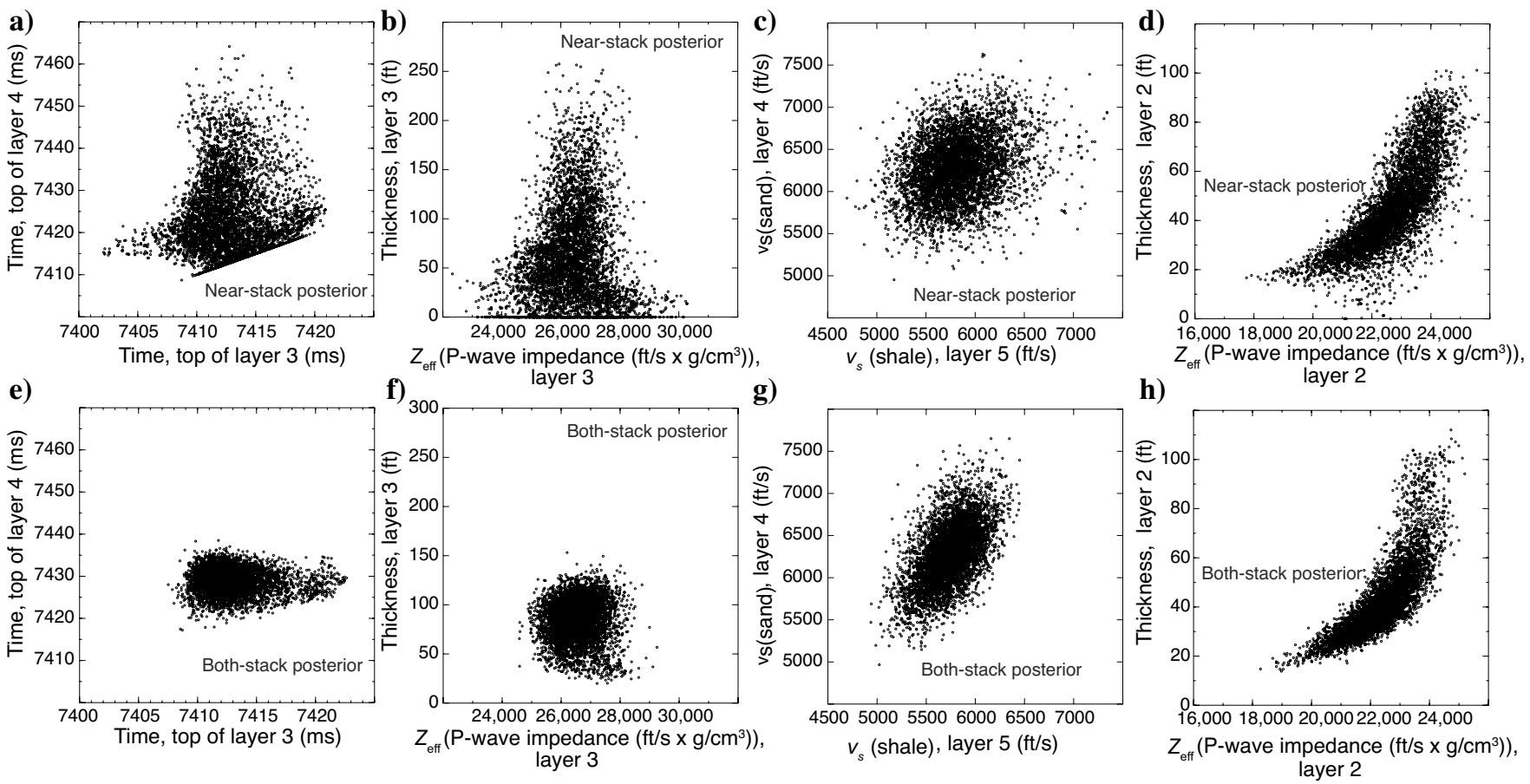

Figure 13. (a-d) Scatterplots of selected property pairs from the near-stack-only inversion. (e-h) The same, using both stacks. (a and b) (Clear tuning-ambiguity effects in the delineation of layer 3 which are not resolved by the use of the near stack only: An appreciable fraction of realizations permit pinchouts in thin layer 3. (e and f) The additional stack resolves this ambiguity. (c and g) Shear velocity $\left(v_{s}\right)$ pair-samples of the layer 4 sand and layer 5 shale, showing how the far stack induces the expected shear velocity correlation across layers. The extra constraint does not significantly reduce the uncertainty in most of the histograms of Figure 12, however. 
Table 1. Prior and posterior standard deviations of selected properties of the reservoir layer (layer 2) from the two-stack inversion of example A. Properties are ranked by decreasing order of sensitivity, as computed from the ratio column (posterior/prior).

\begin{tabular}{cccc}
\hline & $\begin{array}{c}\text { Prior } \\
\text { standard } \\
\text { deviation }\end{array}$ & $\begin{array}{c}\text { Posterior } \\
\text { standard } \\
\text { deviation }\end{array}$ & Ratio \\
\hline Property & 0.044 & 0.0028 & 0.06 \\
R_near & 10.00 & 0.80 & 0.08 \\
layer-time $(\mathrm{ms})$ & 0.027 & 0.0028 & 0.10 \\
R_far & 1900.00 & 1240.00 & 0.65 \\
P-wave impedance $\left(\mathrm{g} / \mathrm{cm}^{3}\right) \times(\mathrm{ft} / \mathrm{s})$ & 565.00 & 372.00 & 0.66 \\
$v_{p}($ sand end member, $\mathrm{ft} / \mathrm{s})$ & 0.04 & 0.03 & 0.75 \\
$\phi$ & 0.065 & 0.05 & 0.77 \\
$\rho_{\text {eff }}\left(\mathrm{g} / \mathrm{cm}^{3}\right)$ & 0.025 & 0.022 & 0.88 \\
$\phi_{\mathrm{flt}}$ & 0.11 & 0.12 & $\approx 1$ \\
$\mathrm{NG}$ & & & \\
\hline
\end{tabular}

\section{CONCLUSION}

The quantitative floating-grain rock-physics model of DeMartini and Glinsky has been incorporated into a model-based Bayesian seismic inversion code to assess the inferability of sorting characteristics from seismic data. Development of the requisite trend models requires careful log and core analysis and some simple nonlinear regression studies. The simple synthetic inversion studies we present are closely based on actual asset data, and show that genuinely improved estimates of the floating-grain or sorting characteristics, plus the reservoir porosity, are possible if the seismic data has sufficient $\mathrm{S} / \mathrm{N}$. For peak $\mathrm{S} / \mathrm{N}$ of about $6: 1$ the examples show about $25 \%$ and $15 \%$ improvements in standard deviations for porosity and floatinggrain fraction, respectively. Moreover, since the posterior distribution of floating grain content is a mixture, the full histogram or marginal is more interesting than the first- and second-order statistics alone: Our test cases are examples where the posterior separates clearly into two clusters of clean and poorly sorted rocks.

The refinements in porosity and floating-grain fraction estimates are a modest fraction of the relative improvements in estimation of the layer impedance. We predict the methodologies of this paper will be useful in inferring sorting characteristics whenever the sorting parameter correlates strongly with impedance, and effects of sorting are in a significantly different direction from that of shale laminations. The first condition is usually satisfied, but the second may vary substantially with local geology. For the cases studied, far-offset data was shown to assist in discrimination of poorly sorted rocks by an extra factor of up to $50 \%$.

The asset in question has some particular challenges associated with a relatively weak reservoir reflection coefficient and complex tuning interference from a hard marl above the reservoir cap, so we regard it as a difficult test case. The inversion techniques for rock quality demonstrated in this paper can be expected to produce sharper posterior updates for data sets free of these particular conspiracies.

\section{ACKNOWLEDGMENTS}

James Gunning gratefully acknowledges generous funding from the BHP Billiton technology program. We thank our reviewers for valuable suggestions which have greatly improved this paper.

\section{APPENDIX A CONVERSION OF REGRESSION FORMULAS}

Approximate conversion to the Gunning and Glinsky (2004) form of the coupled regressions (i.e., equations 9 and 10) from the DeMartini and Glinsky style (equations 6 and 7) can be derived by simple algebra and assumption of independence of errors. The result is

$A_{v_{p}}=a_{p}+b_{p}(1+g) a_{\phi}$

$B_{v_{p}}=b_{p} b_{\phi}(1+g)$,

$D_{v_{p}}=b_{p}\left(1-\frac{1+g}{1-f_{c}}\right)$,

$A_{\phi}=-\frac{a_{p}}{b_{p}(1+g)}$,

$B_{\phi}=\frac{1}{b_{p}(1+g)}$,

$C_{\phi}=-\frac{1}{1+g}$,

$\sigma_{\epsilon_{p}}^{\prime}=\sqrt{\sigma_{\epsilon_{p}}^{2}+\left(b_{p}(1+g)\right)^{2} \sigma_{\epsilon_{\phi}}^{2}}$,

$\sigma_{\epsilon_{\phi}}^{\prime}=\frac{\sigma_{\epsilon_{p}}}{\left|b_{p}(1+g)\right|}$.

\section{APPENDIX B}

\section{IMPLEMENTATION DETAILS}

The inversion code (Gunning and Glinsky, 2004) works with two versions of the model vector $\mathbf{m}$. The vector $\mathbf{m}$ has a fully Gaussian prior, with no truncations or restrictions on values. The physical model vector $\mathbf{m}^{\prime}$, which is used in the forward model and its associated likelihoods (seismic, isopachs) is obtained by applying time orderings and truncations (e.g., of NG or saturations) to $\mathbf{m}$, i.e., $\mathbf{m}^{\prime}$ $=f(\mathbf{m})$, where $f()$ embeds these rules. The truncation effectively induces a prior which, for simple properties like NG, is a mixture of a truncated Gaussian distribution and delta functions at endpoints. 

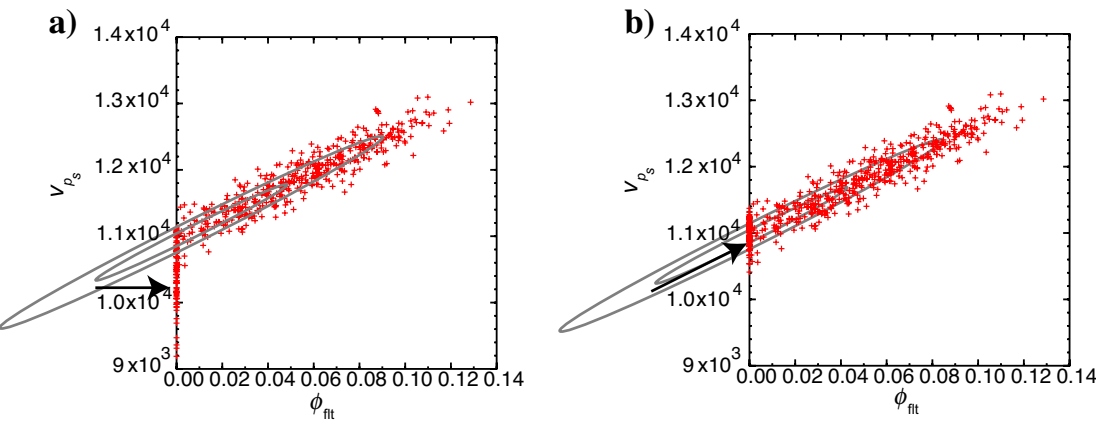

Figure B-1. (a) Pure truncation of $\phi_{\mathrm{flt}}$ resulting in smearing of prior along $\phi_{\mathrm{flt}}=0$ plane. (b) The remappings of equations B-1 through B-4 which seem more reasonable.

\section{REFERENCES}

Abrahamsen, P., A. L. Hektoen, L. Holden, and K. L. Munthe, 1997, Seismic impedance and porosity: Support effects, in E. Y. Baafi and N. A. Schofield, eds., Geostatistics Wollongong '96, Vol. 1, 489-500.

Avseth, P., T. Mukerji, A. Jørtad, G. Mavko, and T. Veggeland, 2001, Seismic reservoir mapping from 3-D AVO in a North Sea turbidite system: Geophysics, 66, 1157-1176.

Avseth, P., T. Mukerji, and G. Mavko, 2005, Quantitative seismic interpretation: Cambridge University Press.

Bachrach, R., and T. Mukerji, 2004, The effect of texture and porosity on seismic reflection amplitude in granular sediments: Theory and examples from a high-resolution shallow seismic experiment: Geophysics, 69, 1513-1520.

Boggs Jr., S., 2006, Principles of sedimentology and stratigraphy: Pearson Prentice Hall.

With the augmented models defined by equations 9 and 10, the linearity means the prior is still Gaussian, but the truncation of $\phi_{\mathrm{flt}}$ in m' must be handled with care. The extra coefficients $D_{v_{p}}, C_{\phi}$ have the effect of placing the prior on inclined ellipsoids in e.g., the $\left\{v_{p}, \phi_{\mathrm{flt}}\right\}$ plane, so pure truncation on $\phi_{\mathrm{flt}}$ has the effect of smearing the tail of the distribution onto the plane $\phi_{\mathrm{flt}}=0$ in a direction off the principal axes. This is clearly an undesirable way to handle the prior. Figure B-1 shows a scatter plot of points produced from a prior constructed in this naive way, with the obvious artifacts. A more reasonable way to handle the truncation is with the mappings (only for $\phi_{\mathrm{flt}}<0$ ):

$$
\begin{aligned}
v_{p}^{\prime} & =v_{p}-D_{v_{p}} \phi_{\mathrm{flt}}, \\
\phi^{\prime} & =\phi+B_{\phi}\left(v_{p}^{\prime}-v_{p}\right)-C_{\phi} \phi_{\mathrm{flt}}, \\
v_{s}^{\prime} & =v_{s}+B_{v_{s}}\left(v_{p}^{\prime}-v_{p}\right), \\
\phi_{\mathrm{flt}}^{\prime} & =0,
\end{aligned}
$$

which forces the remapping to occur along directions parallel to the principal axes.

This mapping minimizes the difference $\left(\mathbf{m}^{\prime}-\mathbf{m}\right)^{T} C_{P}^{-1}\left(\mathbf{m}^{\prime}-\mathbf{m}\right)$, subject to the positivity constraint, which seems a reasonable formulation. The prior will then be a mixture of clean rocks $\left(\phi_{\mathrm{flt}}=0\right)$ and poorly sorted rocks distributed along the ellipsoid with $\phi_{\mathrm{flt}}>0$

Note that the actual Gassman fluid substitution calculation that occurs later in the forward model uses only the pure fluids (oil, gas, etc.), as the Gassman-like effect of the floating-grain presence is implicitly accounted for by the floating-grain terms in the modified regressions.
Buland, A., A. Kolbjornsen, and H. Omre, 2003, Rapid spatially coupled AVO inversion in the Fourier domain: Geophysics, 68, 824-836.

Buland, A., and H. Omre, 2003, Bayesian linearized AVO inversion: Geophysics, 68, 185-198.

Coleou, T., F. Allo, R. Bornard, J. Hamman, and D. Caldwell, 2005, Petrophysical seismic inversion: 75th Annual International Meeting, SEG, Expanded Abstracts, 1355-1359.

DeMartini, D. C., and M. Glinsky, 2006, A model for variation of velocity versus density trends in porous sedimentary rocks: Journal of Applied Physics, 100, 014910.

Dutta, T., T. Mukerji, G. Mavko, and P. Avseth, 2006, Reservoir quality prediction by integrating sequence stratigraphy and rock physics: 76th Annual International Meeting, SEG, Expanded Abstracts, 1811-1815.

Dvorkin, J., and M. A. Gutierrez, 2002, Grain sorting, porosity, and elasticity: Petrophysics, 43, 185-196.

Dvorkin, J., and A. Nur, 1996, Elasticity of highporosity sandstones: Theory for two North Sea datasets: Geophysics, 61, 1363-1370.

Eidsvik, J., P. Avseth, H. Omre, T. Mukerji, and G. Mavko, 2004, Stochastic reservoir characterization using prestack seismic data: Geophysics, 69 , 978-993.

Fraley, C., and A. E. Raftery, 2003, Enhanced model-based clustering, density estimation, and discriminant analysis software: MCLUST: Journal of Classification, 20, 263-286.

Gunning, J., and M. Glinsky, 2004, Delivery: An open-source model-based Bayesian seismic inversion program: Computers and Geosciences, 30, 619-636.

Marion, D. P., 1990, Acoustical, mechanical, and transport properties of sediments and granular materials: Ph.D. thesis, Stanford University.

Mavko, G., T. Mukerji, and J. Dvorkin, 1998, The rock physics handbook: Cambridge University Press.

McManus, J., 1988, Grain size determination and interpretation, in M. Tucker, ed., Techniques in sedimentology: Blackwell Science Publications, $63-85$.

Nur, A., D. Marion, and H. Yin, 1991, Wave velocities in sediments, in J. M. Hovem, M. D. Richardson, and R. D. Stoll, eds., Shear waves in marine sediments: Kluwer Academic Publishers, 131-140.

Vernik, L., 1997, Predicting porosity from acoustic velocities in siliciclastics: Anew look: Geophysics, 62, 118-128. 